Research Paper

\title{
In Vitro Evaluation of Theranostic Polymeric Micelles for Imaging and Drug Delivery in Cancer
}

\author{
Rajiv Kumar $^{\bowtie}$, Apurva Kulkarni, Dattatri K Nagesha, Srinivas Sridhar \\ Electronic Materials Research Institute, Northeastern University, Boston MA USA. \\ $\triangle$ Corresponding author: E-mail: r.kumar@neu.edu. \\ (C) Ivyspring International Publisher. This is an open-access article distributed under the terms of the Creative Commons License (http://creativecommons.org/ \\ licenses/by-nc-nd/3.0/). Reproduction is permitted for personal, noncommercial use, provided that the article is in whole, unmodified, and properly cited.
}

Received: 2011.12.07; Accepted: 2012.01.14; Published: 2012.07.31

\begin{abstract}
For the past decade engineered nanoplatforms have seen a momentous progress in developing a multimodal theranostic formulation which can be simultaneously used for imaging and therapy. In this report we describe the synthesis and application of theranostic phospholipid based polymeric micelles for optical fluorescence imaging and controlled drug delivery. CdSe quantum dots (QDs) and anti-cancer drug, doxorubicin (Dox), were co-encapsulated into the hydrophobic core of the micelles. The micelles are characterized using optical spectroscopy for characteristic absorbance and fluorescence features of QDs and Dox. TEM and DLS studies yielded a size of $<50 \mathrm{~nm}$ for the micellar formulations with very narrow size distribution. A sustained release of the drug was observed from the co-encapsulated micellar formulation. In vitro optical fluorescence imaging and cytotoxicity studies with HeLa cell line demonstrated the potential of these micellar systems as efficient optical imaging and therapeutic probes.
\end{abstract}

Key words: Theranostic Polymeric Micelles, Imaging, Drug Delivery, Cancer

\section{Introduction}

Translational nanotechnology has shown a great potential in different biomedical applications including cancer management. [1-3] The early inroads in the development of these theranostic platforms provides a highly versatile and valuable components for building up conceptual 'personalized medicine' which emphasizes on the tailored treatment based on the individual patient's biology for the final treatment outcome. Generally, the application of these nanoplatforms can be summarized into therapy and diagnosis, hence 'Theranostic'. [4-6] The successful development of these theranostic nanoplatforms requires concomitant development of quantitative imaging modalities that not only allow early detection of the disease but also reliably measure therapeutic response. In this regard, nanocarriers offer the potential to develop novel imaging based platforms that could potentially integrate diagnostic and therapeutic applications in oncology. In addition, the inclusion of imaging agents would provide a realtime, non-invasive assessment of tumor physiology, drug delivery and therapeutic response. [7]

The quest for building a theranostic platform has resulted in a number of nanoparticles based formulations which includes inorganic nanoparticles, liposomes, micelles, emulsions, dendrimers etc. [8-13] Polymeric micelles provide an attractive platform in which the formulation can be designed with multiple imaging modalities along with a drug payload. Phospholipid based polymeric micelles is one such system that have shown a great potential in delivering hydrophobic drugs and have been studied extensively 
because of their attractive features to fulfill the requirements for selective drug delivery. [14] Polymeric micelles are self-assembled amphiphilic copolymers in the form of core/shell nanostructures. The hydrophilic part of the copolymer usually forms the shell and provides stability in the aqueous medium. The hydrophobic core of the micelles has been used extensively for encapsulation of various non-polar drugs like paclitaxel, doxorubicin, amphotericin-B. [15-17] The hydrophobic core of the micelles regulates the release of the encapsulated hydrophobic drug in a sustained manner whereas the hydrophilic polyethylene glycol (PEG) chains on the surface of the micelles impart excellent colloidal stability in aqueous as well as in vivo environment. In most formulations, the hydrophilic chain consists of PEG units. In systemic circulation the hydrophilic PEG chains prevents the binding of the serum proteins (opsonization) on the micellar surface and thus avoids rapid clearance from the blood stream by macrophages. The presence of PEG coatings and small size of the micelles results in a very efficient passive accumulation of these micelles in the tumor by the well-known Enhanced Permeability and Retention (EPR) effect. [18]

Here we report an efficient theranostic phospholipid based micellar system encapsulating anti-cancer drug doxorubicin (Dox: for therapy) and CdSe quantum dots (QDs: diagnosis). Fluorescent QDs have been investigated extensively as luminescent biological probe because of their nanometer dimensions and attractive optical properties including high-absorption cross section, narrow and symmetric emission band and high resistance to photobleaching. [19] The hydrophobic QDs can be easily encapsulated in the core of the micelles because of the ultra-small dimension. The anti-cancer drug Dox has been studied extensively in a variety of cancer models. [20, 21] The cytotoxic mechanism of the Dox involves DNA intercalation as well as by lipid peroxidation and inhibition of the topoisomerase II. [22, 23] In our micellar formulation we have combined QDs and Dox for building a robust theranostic platform. In earlier reports, similar approaches by conjugating the QDs and drugs were used. [24, 25] In this manuscript, we have exploited the hydrophobic core of the DSPE-PEG based micelles for encapsulation of Dox and QDs together. The release kinetics was carried out to confirm the sustained release of the Dox from the micelles. The therapeutic efficacy of the micellar formulation was tested in vitro using HeLa cell line. In vitro imaging studies were also performed for the cellular uptake behavior of the micelles. The multimodality of the micellar formulation with the presence of PEG for passive accumulation, high colloidal stability and therapeutic efficacy makes these polymeric micelles potential candidate for diagnosis and therapy of cancer.

\section{Materials and Methods}

\section{Materials}

Lipids, 1,2-distearoyl-sn-glycero-3-phosphoethanolamine-N-[methoxy(polyethylene glycol)-2000] (DSPE-mPEG-2000), 1,2-distearoylglycero-3-phosphocholine (DSPC), in powdered form were procured from Avanti Polar Lipids (Alabaster, AL). Doxorubicin hydrochloride (Dox.HCl), chloroform and other solvents were purchased from Sigma and were used without further purification. CdSe QDs (Lumidot 480 $\mathrm{nm}$, hexadecylamine stabilized dispersed in toluene with a of size $2.1 \mathrm{~nm}$ at a concentration of $5 \mathrm{mg} / \mathrm{ml}$ ) were purchased from Sigma. HPLC grade water was used in the formulations. HeLa cell line was procured from ATCC (Rockville, MD) and cultured according to instructions supplied by the vendor. MTS reagent CellTiter 96® AQueous One Solution was procured from Promega (USA). Biological growth medium, DMEM, and serum were purchased from Invitrogen. Microfuge membrane-filters (NANOSEP 100K OMEGA) are a product of Pall Corporation.

\section{Methods}

\section{Modification of Doxorubicin}

The hydrophilic form of the drug Doxorubicin $\mathrm{HCl}$ was converted to its hydrophobic form by neutralizing the hydrochloride salt with the base triethylamine. Briefly, to $10 \mathrm{mg}$ of powdered Dox. $\mathrm{HCl}$, $0.54 \mathrm{mmol}$ of triethylamine was added and incubated overnight. The non-aqueous from of the Dox was extracted using methylene chloride and evaporated. [26] The powdered Dox was dissolved in chloroform at a conc. of $2 \mathrm{mg} / \mathrm{ml}$ and stored at $4^{\circ} \mathrm{C}$ until further use.

\section{Synthesis of polymeric micelles encapsulating QDs and Doxorubicin:}

Polymeric micelles encapsulating Dox was prepared using our previously reported method with slight modifications. [13] Briefly, a total of $5.5 \times 10^{-6}$ mol of phospholipid mixture dispersed in $1 \mathrm{ml}$ of chloroform was used for making the polymeric micelles. The composition of the different lipids in the mixture contained 40\% DSPE-PEG and 60\% DSPC. For preparing the micelles, $50 \mu \mathrm{l}$ of Dox $(2 \mathrm{mg} / \mathrm{ml}$ in chloroform) and $200 \mu \mathrm{l}$ of CdSe $(5 \mathrm{mg} / \mathrm{ml}$ in toluene) were taken in a round bottom flask followed by the addition of $1 \mathrm{ml}$ of prepared phospholipid solution as described above. The solution was sonicated for $15 \mathrm{sec}$ followed by evaporation of the chloroform using a 
rotatory evaporator. The obtained thin film was heated over a water bath for $2 \mathrm{~min}$ at $70^{\circ} \mathrm{C}$ and finally rehydrated with $2 \mathrm{ml}$ HPLC grade water. The solution was further sonicated for $10 \mathrm{~min}$ to obtain an optically transparent solution that contains Dox and QDs encapsulated polymeric micelles. The non encapsulated QDs were removed by centrifuging the samples at $2000 \mathrm{rpm}$ for $10 \mathrm{~min}$. Since the non encapsulated QDs were not stable in aqueous environment, they tend to aggregate and can be easily separated at low centrifuge speed. The non-encapsulated Dox was removed from the micellar suspension by using $100 \mathrm{kDa}$ centrifuge spin filters. The pellet was further dispersed in $2 \mathrm{ml}$ of water and stored at $4^{\circ} \mathrm{C}$ for further use.

Characterization of polymeric micelles: UV-Visible Absorbance and Photoluminescence (PL) spectroscopy

The optical studies for Dox, QDs and in their encapsulated forms were done by absorbance and fluorescence measurements. The absorption spectra were collected using a Agilent 8453 UV-Vis spectrophotometer over a wavelength range from 300 to 800 $\mathrm{nm}$. All samples were used as prepared and loaded into a quartz cell for measurements in the appropriate solvent and measured against water or chloroform as the reference. The emission spectra were collected using a Fluoromax 4 Spectrofluorometer (Jobin Yvon; fluorescence spectra) using an excitation wavelength of $405 \mathrm{~nm}$ (QDs) and $488 \mathrm{~nm}$ (Dox).

\section{Transmission Electron Microscopy (TEM)}

Transmission Electron Microscopy (TEM) images were obtained using a JEOL model JEM- 100CX microscope at an acceleration voltage of $80 \mathrm{kV}$. The specimens were prepared by drop-coating the sample dispersion onto an amorphous carbon coated 300 mesh copper grid followed by addition of another drop of 1\% phosphotungstic acid (for negative staining) which was neutralized with dilute $\mathrm{NaOH}$ solution to maintain the $\mathrm{pH} 7$. The grid was placed on a filter paper to absorb excess solvent.

\section{Particle Size Measurements}

Dynamic Light Scattering measurements were done by using 90Plus zeta sizer (Brookhaven Inc, NY) for measuring the hydrodynamic diameter of the different polymeric micelles formulations by taking a diluted sample in $3 \mathrm{ml}$ cuvette. Zeta potential measurements were also done using the same instrument.

\section{Release Kinetics Studies}

The release kinetic studies were carried out by incubating the QD-Dox formulation at $37^{\circ} \mathrm{C}$ with $1 \%$ tween 80 solution in water. The concentration of Dox in the QD-Dox micelles was $46 \mu \mathrm{g} / \mathrm{ml}$ of micellar so- lution. The samples were spin filtered after a specified period of time using microfuge membrane-filter (NANOSEP 100K OMEGA, Pall Corporation, USA) at $14,000 \mathrm{rpm}$ for $30 \mathrm{~min}$ (spin-filtration). The Tween-80 micelles along with any released Dox flowed through this membrane and fluorescence was measured for each filtrate collected. For estimating the released drug from the micelles, a standard curve was made by measuring the fluorescence with different concentration of the Dox and then reading out the released dox concentration from the standard curve and finally plotting the cumulative release against the time. The percentage of the released drug was calculated by taking into account the total drug encapsulated in the micelles and amount released at each time points.

\section{Cell Staining Studies}

For in vitro imaging with different polymeric micelles formulations, human cervical cancer cell line HeLa was cultured in Dulbecco minimum essential media (DMEM) with 10\% fetal bovine serum (FBS), $1 \%$ penicillin, and $1 \%$ amphotericin $B$. The day before micelles treatment, cells were seeded in $35 \mathrm{~mm}$ culture dishes. On the treatment day, the cells, at a confluency of $70-80 \%$ in serum-supplemented media, were treated with the polymeric micelles at a specific concentration (100 $\mu \mathrm{l} / 1 \mathrm{ml}$ media) for two hours at $37^{\circ} \mathrm{C}$. The cells were washed twice with DMEM before imaging to remove any free micelles present in the culture plates. The cells were imaged using a confocal microscope after 2 and $24 \mathrm{hr}$ incubation time period with the micelles.

\section{Confocal Microscopy}

Confocal Microscopy images were obtained using a Zeiss LSM 700 confocal microscope (Carl Zeiss, Europe) with excitation source at $405 \mathrm{~nm}$ and $488 \mathrm{~nm}$ for QDs and Dox respectively. For the QDs and Dox formulations two separate emission channels were used for sequential emission acquisition of two different fluorophores. All images were taken under exact same conditions of laser power, aperture, gain, offset, scanning speed, and scanning area.

\section{In vitro Cytotoxicity Assays}

HeLa cells were dispensed into a 96-well flat-bottom microtiter plate (Nunc) (5000 cells/well) using DMEM medium with 10\% FBS and allowed to attach overnight. Cell viability was assessed by the CellTiter 96® AQueous One Solution Cell Proliferation Assay. This assay is a colorimetric method to determine the number of viable cells in proliferation or cytotoxicity assays and is based on the bioreduction of MTS tetrazolium compound (Owen's reagent) by the 
cells into a colored formazan product that is soluble in tissue culture medium. This conversion is presumably accomplished by NADPH or NADH produced by dehydrogenase enzymes in metabolically active cells In brief, after $4 \mathrm{~h}$ treatment with different micellar formulations and free Dox, media was changed, and the AQueous One Solution was added to the cells. Each was mixed for $2 \mathrm{~min}$ and allowed to incubate for $4 \mathrm{hrs}$ inside incubator. Absorbance was measured at $490 \mathrm{~nm}$ using a microplate reader (Bio-Tek Synergy HT microplate reader) and data were expressed as a percentage of the control. Tests were performed in quadruplicate. Each point represents the mean $\pm S D$ (bars) of replicates from one representative experiment.

\section{Results}

The micellar formulation encapsulating hydrophobic QDs and Dox were prepared by lipid hydration method. The hydrophobic core resulting from the bulky acyl chains of the phospholipid provides space for the hydrophobic compounds as illustrated in Figure S1/Scheme 1. This hydrophobic core is further stabilized in the aqueous environment by PEG chains. This amphiphilic nature of the phospholipids results in a stabilization of the hydrophobic compounds in the aqueous environment making delivery of many of these hydrophobic drugs possible. [27, 28] Highly fluorescent QDs are generally synthesized with hydrophobic ligands and for biological applications have to be converted to hydrophilic particles. There- fore, the process of micellar encapsulation of QDs provides a simple and robust platform in which the hydrophobic compounds can be co-delivered avoiding the complicated methods for ligand displacement and further functionalization to render hydrophobic particles biocompatible and stable in in vitro and in vivo systems.

Figure 1 shows the size distribution profile of various micellar formulations. As it can be seen from the figure, both the micellar formulations, QDs micelles and QD-Dox Micelles showed a hydrodynamic diameter of approx $35 \mathrm{~nm}$ and $50 \mathrm{~nm}$ respectively, which is typical for the micellar system composed of mixture of lipids. The slight increase in the size of the QD-Dox micelles can be attributed to the additional swelling of the micelles because of the addition of Dox. Representative TEM image of these micelles co-encapsulating QD-Dox showed almost spherical morphology with a uniform size distribution of 50 $\mathrm{nm}$. The cluster of QDs in discrete pockets shows the encapsulation of the QDs in the micelles core (figure 1: right panel A). TEM image of QDs dispersed in toluene (figure 1: right panel B) showed a narrow distribution of the individual QDs with a size $\sim 2.2 \mathrm{~nm}$. It is worth noting that in case of QD-Dox micelles, multiple QDs are localized in the hydrophobic core of the micelles as can be visualized from the figure. Depending upon the ratio of phospholipids to the hydrophobic compounds, the core of the micelles can be tuned to accommodate multiple to single hydrophobic molecules/nanoparticles.

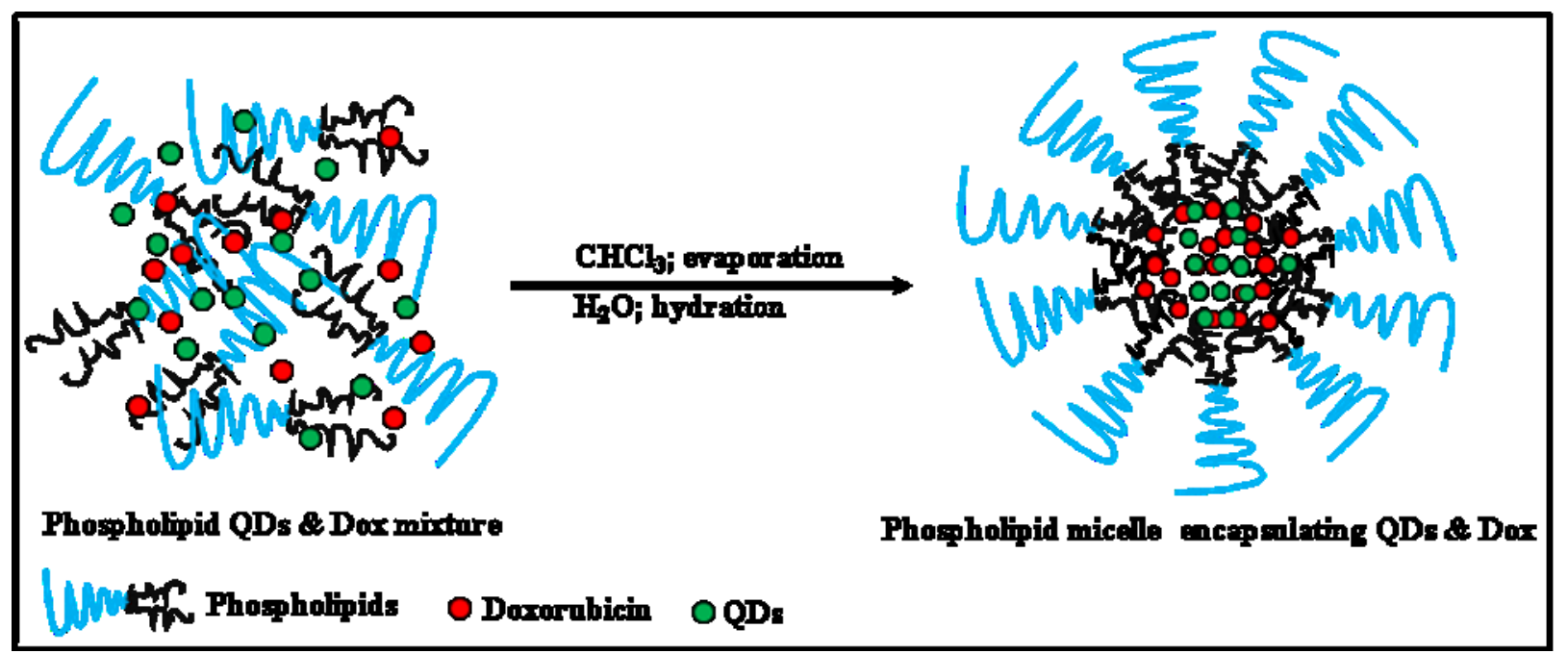

Figure SI. (Scheme I) Schematic representation of the synthetic strategy of the phospholipid based micelles encapsulating CdSe QDs and doxorubicin. 

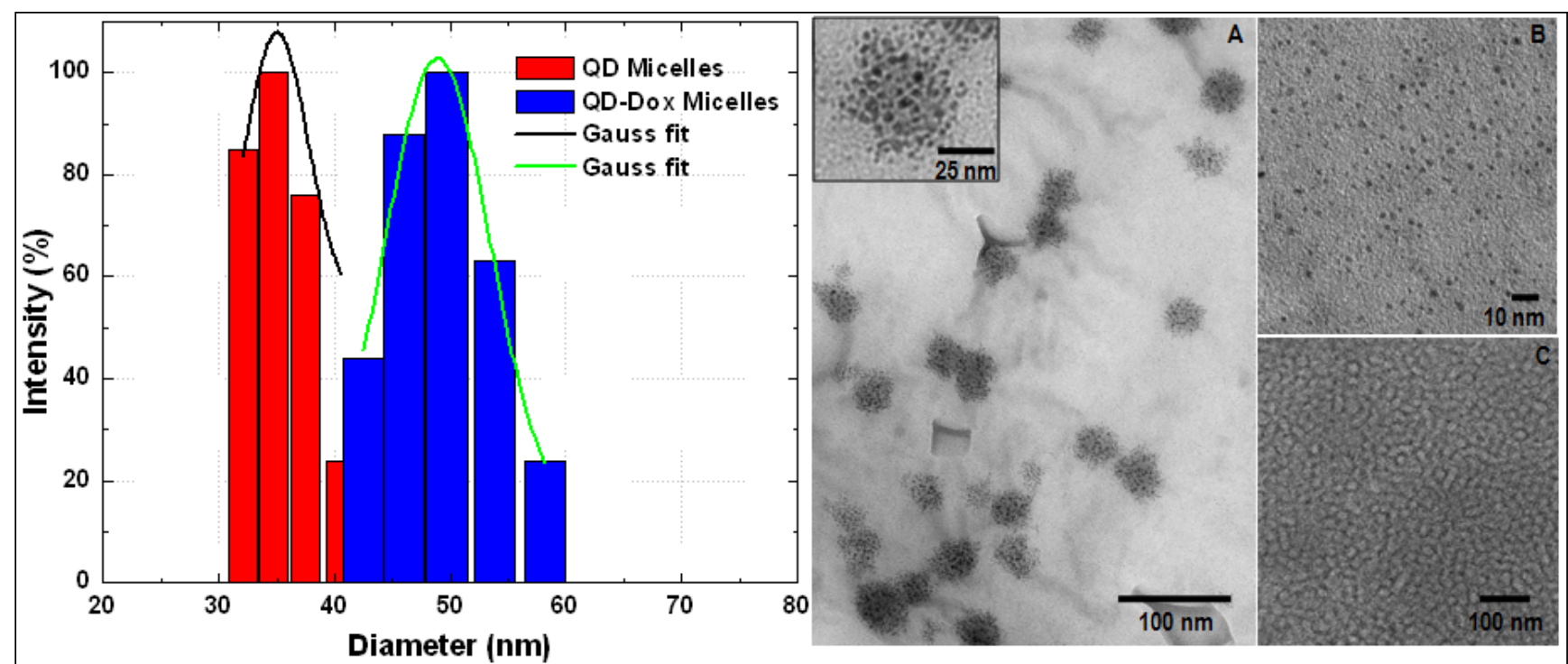

Figure I: Size distribution and morphology studies of the micellar formulations by Dynamic light scattering measurements (left panel) and Transmission electron Microscopy (right panel). TEM image of (A) QD-Dox micelles, (B) QDs dispersed in toluene and (C) blank micelles. Inset shows the magnified image of the QD-Dox micelles.

In earlier studies by Dubertret et al have shown the encapsulation of single QD in the core of the phospholipid micelles whereas Park et al have shown the number of iron oxide encapsulated in the core of the micelles can be increased by changing the ratio of nanoparticles and phosphoilipids. [29,30] We have also studied the surface charge of these micelles by measuring their zeta-potential values. Both the micellar formulations showed a net negative charge of $-31 \mathrm{mV}$ which can be to the presence of PEG and partial negative charge from the lipid DSPC on the surface of the micelles.

Figure 2 and 3 shows the optical characterization of the micellar formulations. As prepared samples with a concentration of $46 \mu \mathrm{g}$ Dox and $500 \mu \mathrm{g}$ of QDs in QD-Dox micelles and $500 \mu \mathrm{g}$ of QDs in QD micelles dispersed in $2 \mathrm{ml}$ of water were used for measurements. Figure 2 shows the absorption spectra of the QDs and Dox in free as well as encapsulated form. The QDs spectra demonstrate an absorption feature at $\sim 460 \mathrm{~nm}$. The absorption peak for the QD-Dox sample was merged with the QDs absorption peak and appears as a small hump in the $500-600 \mathrm{~nm}$ range. The absorption spectral feature of Dox in micellar formulation is very small due to the low concentration of the Dox and sensitivity of the instrument. Free Dox in chloroform showed a typical Dox optical feature with $\lambda_{\max }$ centered around $490 \mathrm{~nm}$.

The photoluminescence studies with the free QDs showed an emission band at $\sim 478 \mathrm{~nm}$ whereas in the micellar form the emission band is slightly blue shifted to $470 \mathrm{~nm}$ (Figure 3). QDs-Dox micellar formulation showed an intense Dox fluorescence with typical emission band at $590 \mathrm{~nm}$. [15] The Dox spectrum retains the typical molecular Dox fluorescence characteristic feature. The QDs peak for the QD-Dox micelles has been enlarged to show the QDs emission from the co-encapsulated micellar system.

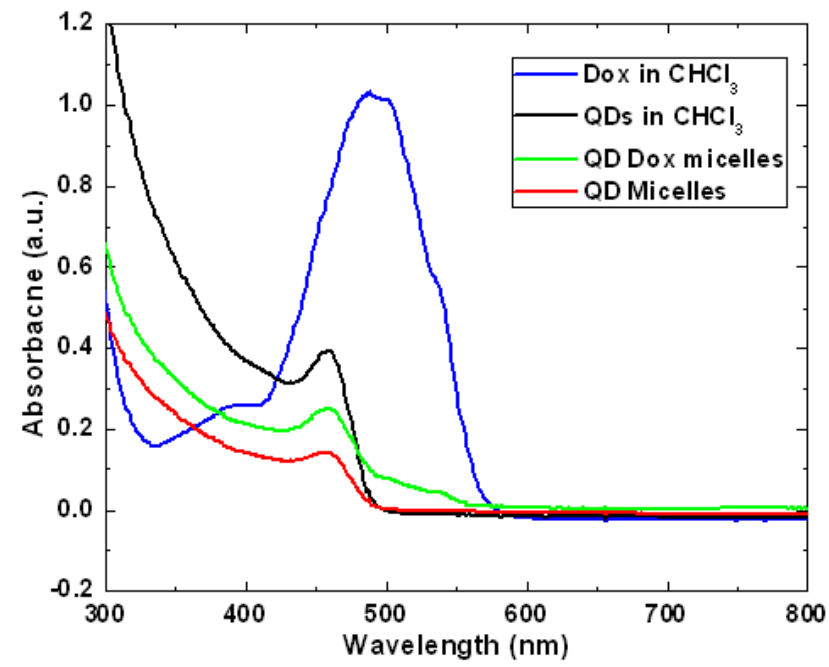

Figure 2: UV-Visible absorbance spectra of CdSe QDs and doxorubicin in free form in chloroform as well as in encapsulated form in micelles. 


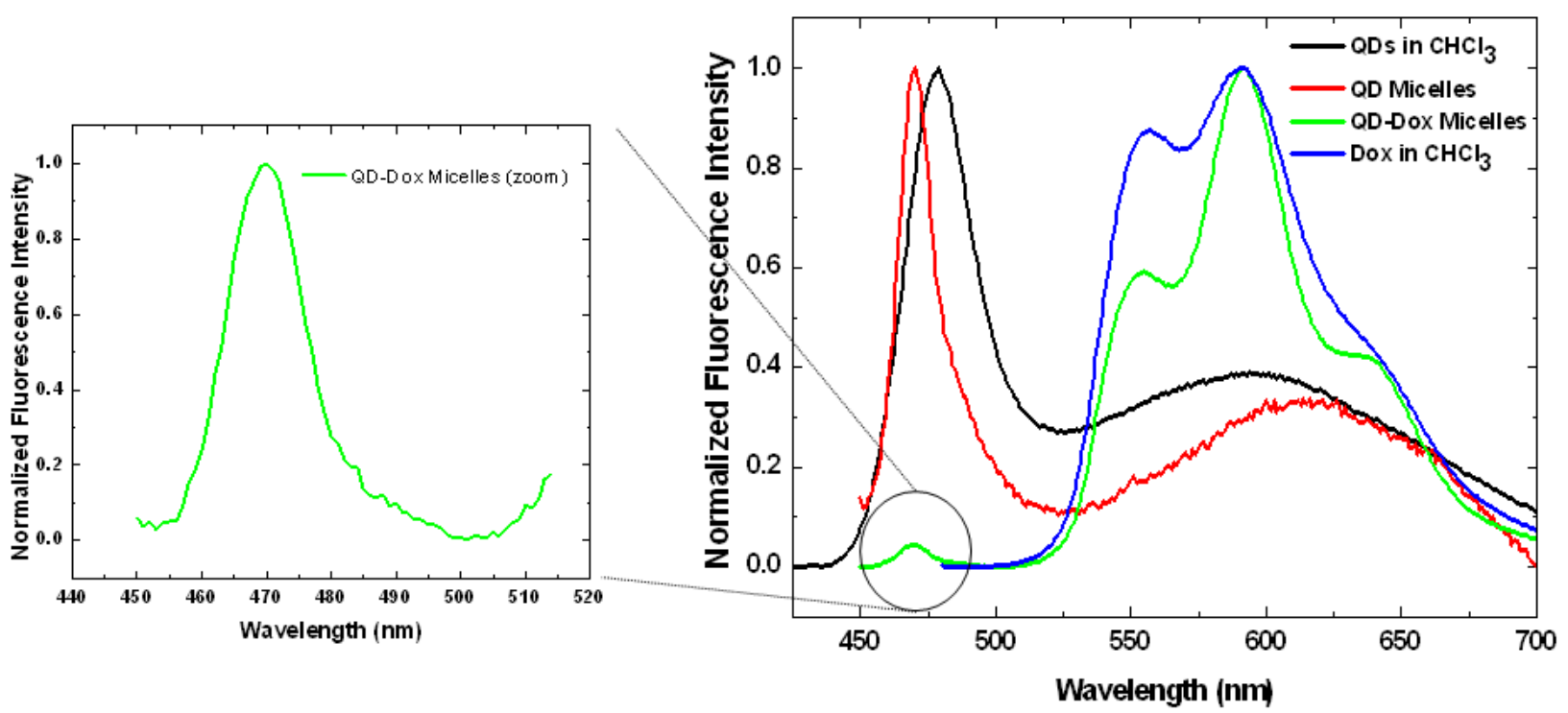

Figure 3: Photoluminescence spectra of the CdSe QDs and doxorubicin in free form in chloroform as well as in encapsulated form in micelles. The spectrum of QDs in QD-Dox micelles is enlarged on the left for clarity. (See supporting info for non-normalized data).

The photoluminescence spectrum of free QDs in Figure 3 shows two distinct fluorescence peaks. The first peak at $\sim 470 \mathrm{~nm}$ corresponds to excitonic band emission and is slightly red-shifted compared to the absorption peak at $\sim 460 \mathrm{~nm}$. The second broad peak centered at around $600 \mathrm{~nm}$ is attributed to deep trap emission and is typically seen at longer wavelengths compared to the narrow and intense excitonic emission peak. The deep trap emission arises due to the presence of deep trap sites on the surface of quantum dots that is associated with chalcogen vacancies. It is an inherent property in a QD that is incorporated during the synthesis. This phenomenon is most prominent in small sized quantum dots that have greater probability of chalcogen vacancies due to the large surface to volume ratio. [32-34] The QDs used in this experiment were $\sim 2.1 \mathrm{~nm}$ and hence have the broad deep trap emission peak. Upon encapsulation within micelles, there is some degree of aggregation resulting in the deep trap emission peak to shift from $600 \mathrm{~nm}$ to $\sim 640 \mathrm{~nm}$.

The release of the encapsulated drug from the micelles was studied by fluorescence spectroscopic measurements. Figure 4 presents the release kinetic profile of the encapsulated Dox from the QD-Dox micelles. The results showed a sustained release of the Dox from the micelles over a period of 7 days. Almost $95 \%$ of the cumulative drug release was observed from the micelles within 7 days. The release of the drug from the micelles can be modulated, if needed, by adding cholesterol to the micellar formulation which imparts rigidity and reduces the fluidity of micellar structure and thus slowing down the release of the encapsulated drug. However, in this system, $95 \%$ drug release over 7 days is beneficial as both the drug and QDs can be cleared out of the body faster upon drug release and destabilization of the micelles. For carrying out the release kinetic studies, it is worth noting that the use of serum as external media would have been the best, however using the dialysis method; the cellulose dialysis membrane is completely incompatible with the serum. Thus we have used 1\% Tween 80 which mimics the in vivo serum conditions by forming hydrophobic pockets which can stabilize the released drug from the micelles and thus avoids aggregation of the hydrophobic drug in the aqueous environment.

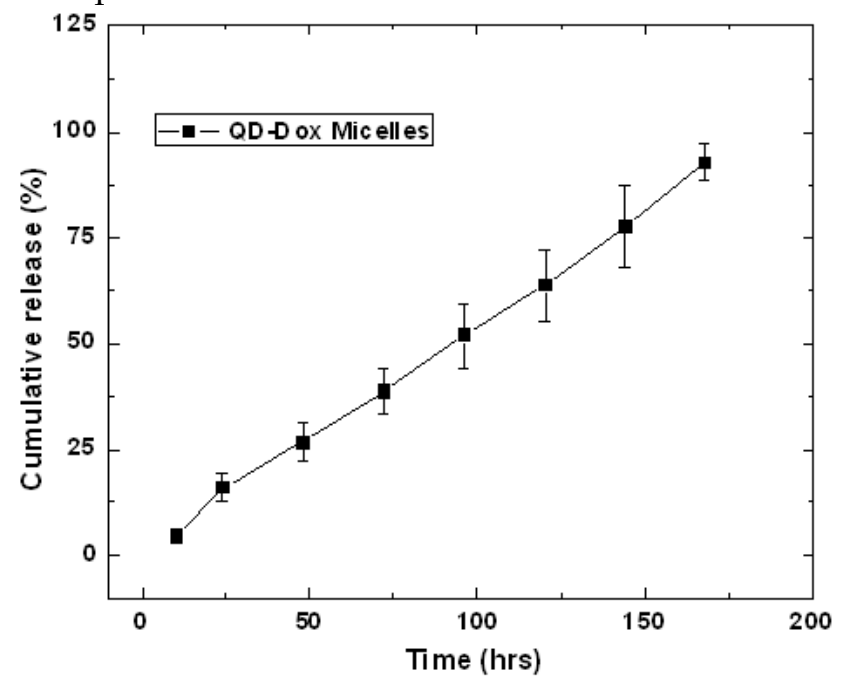

Figure 4: Release kinetics study for estimating the release of the doxorubicin from the QD-Dox micelles. 
To verify the imaging capabilities of the encapsulated QDs, we have carried out in vitro cellular uptake studies using human cervical cancer cell line (HeLa) and studying the uptake by confocal microscopy. Figure 5 shows the in vitro confocal images of the HeLa cells incubated with QD micelles for two different time points of $2 \mathrm{hrs}$ and $24 \mathrm{hrs}$. From the figure a time dependent increased uptake of the QDs is quite evident. A robust uptake of the QDs can be visualized clearly over a period of $24 \mathrm{hrs}$. The accumulation of QDs has been seen mostly centered around the endoplasmic region of the cells, which is consistent with pattern of nanoparticles uptake as shown earlier. [35] Figure 6 shows the confocal fluorescence images of the HeLa cells treated with QD-Dox micelles. Dox has been known to penetrate the cell nucleus where it intercalated with cellular DNA to show cytotoxicity.

Within 2 hrs a strong accumulation of Dox was observed from the nucleus of HeLa cells. The QDs fluorescence signal remained similar to what was observed with the QD micelles after 2hrs incubation time. The overlaid images clearly showed the expected distribution of the QD-Dox micelles with Dox accumulating in the nucleus and QDs in cytoplasm as well as plasma membrane. Incubation of QD-Dox micelles for $24 \mathrm{hrs}$ resulted in a higher cytotoxicity as a result the cells were not imaged after 24 hrs incubation.
The use of cadmium based QDs usually raises the concern for potential cytotoxicity. We have further carried out in vitro cytotoxicity assays to confirm the biocompatibility of the QDs micelles as well as for estimating the therapeutic efficacy of the encapsulated Dox. Figure 7 shows the results obtained from the cell viability assay (MTS assay) carried out with HeLa cell line incubated with QD micelles, free Dox and QD-Dox micelles. The concentration of all the samples was calculated based on the concentration of Dox present in the micelles. The results clearly demonstrated the potential of these theranostic micelles. QD micelles showed $>95 \%$ cell survival after 24 hrs incubation time period demonstrating biocompatibility of the QD micelles. QD-Dox micelles at a Dox concentration of $5 \mu \mathrm{g} / \mathrm{ml}$ showed a strong cytotoxic effect ( $50 \%$ cell survival) whereas at the same concentration, free Dox showed more than $65 \%$ cell survival. This result clearly depicts the efficient delivery of the Dox to the HeLa cells. The sustained release of the drug resulted in a constant pool of drug which is available for the cells for longer time period as opposed to free drug. Based on the above results we believe that these stable and robust theranostic micellar formulation co-encapsulating QDs and Dox can be used as an efficient nanoplatform for imaging and therapy of cancer in vitro and in vivo. However, further in vivo studies are required to confirm this.

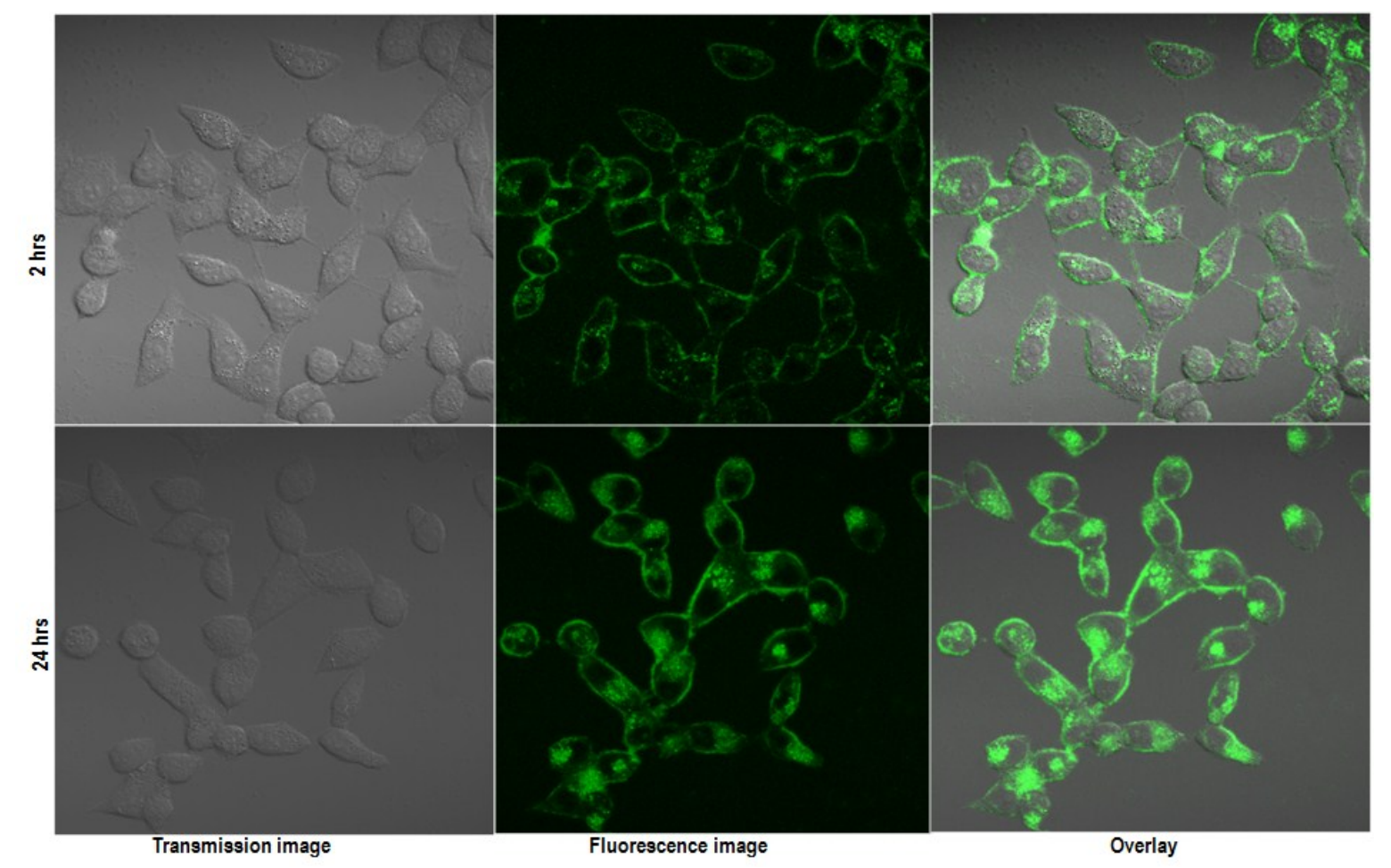

Figure 5: Confocal images of the HeLa cells incubated with QD micelles for 2 hrs (upper panel) and 24 hrs (lower panel). Same instrument settings were used to acquire all the images. 


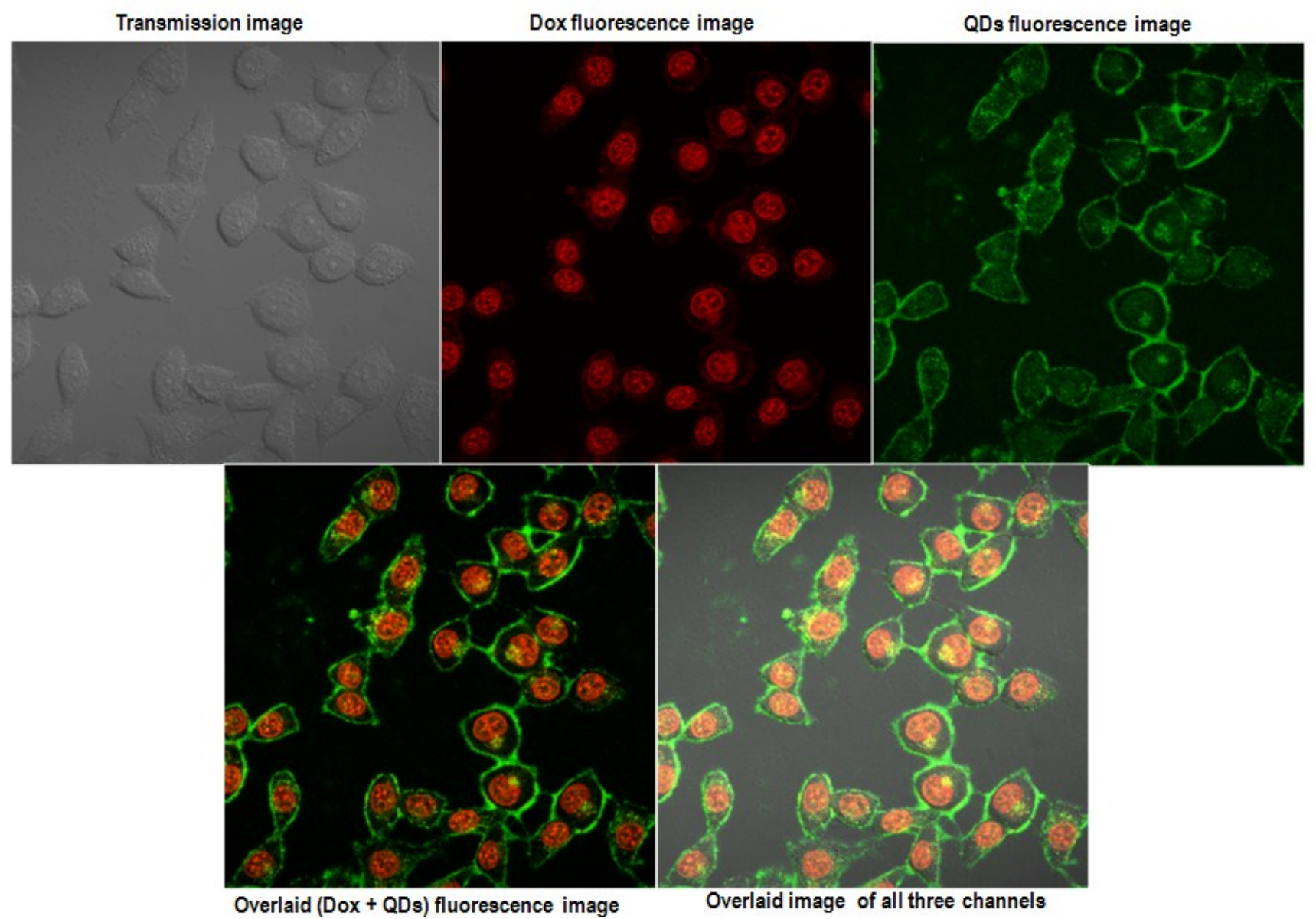

Figure 6: Confocal images of the HeLa cells incubated with QD-Dox micelles for $2 \mathrm{hrs}$. The upper panel shows the images acquired from the three different channels and the lower panel shows the overlaid images.

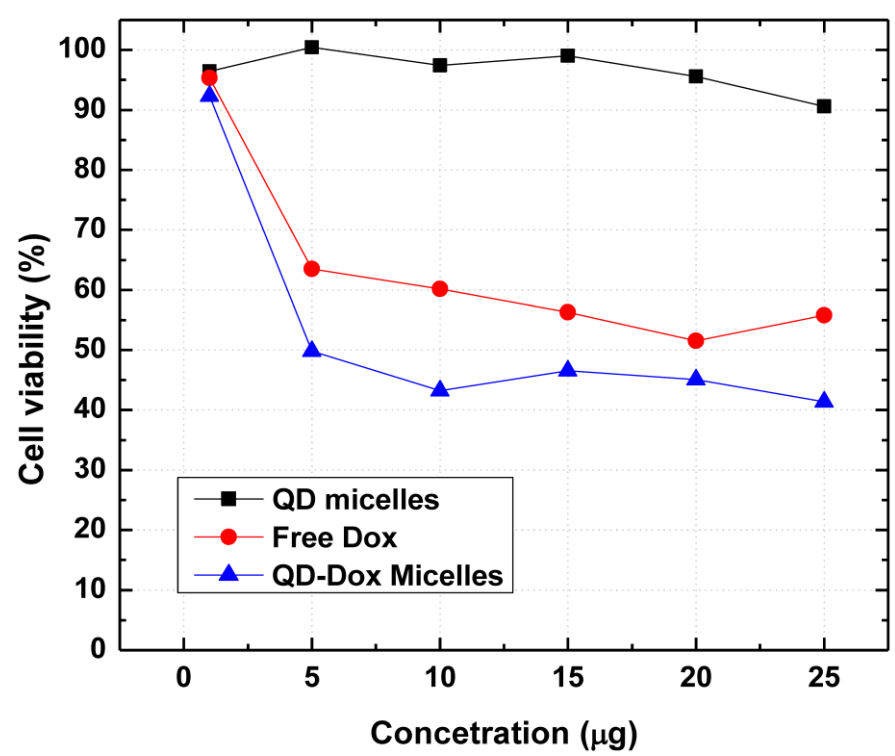

Figure 7: Cytotoxicity assay (MTS assay) carried out with different micellar formulations of QD micelles, QD-Dox micelles and free Dox in HeLa cell line. All the formulations were incubated for $24 \mathrm{hrs}$. 


\section{Conclusions}

In summary, we have synthesized phospholipid based micellar system encapsulating QDs and doxorubicin using a simple lipid hydration method. The encapsulated drug showed a sustained release from the micellar core over a period of 7 days. A strong time dependent uptake of the micelles have been demonstrated successfully using confocal microscopy. The biocompatibility of the micellar system was confirmed by cell viability assay which showed a strong potential of these theranostic micelles for imaging and therapy of different diseased conditions including cancer management.

\section{Competing Interests}

The authors have declared that no competing interest exists.

\section{References}

1. Wagner V, Dullaart A, Bock A. The emerging nanomedicine landscape. Nat Biotechnol. 2006; 24: 1211-8

2. Lim WT, Tan EH, Toh CK, et al. Phase I pharmacokinetic study of a weekly liposomal paclitaxel formulation (Genexol-PM) in patients with solid tumors. Ann Oncol 2009; 21: 382-8.

3. Davis ME, Zuckerman JE, Choi CHJ, et al. Evidence of RNAi in humans from systemically administered siRNA via targeted nanoparticles. Nature. 2010; 464: 1067-0.

4. Sumer B, Gao J. Theranostic nanomedicine for cancer. Nanomedicine. 2008; 3: 137-0.

5. Zhang L, Gu F, Chan J, et al. Nanoparticles in medicine: therapeutic applications and developments. Clin Pharmacol Ther. 2007; 83: 761-9.

6. Papadopoulos N, Kinzler KW, Vogelstein B. The role of companion diagnostics in the development and use of mutation-targeted cancer therapies. Nat Biotechnol. 2006; 24: 985-5.

7. Liong $\mathrm{M}, \mathrm{Lu} \mathrm{J}$, Kovochich $\mathrm{M}, \mathrm{Xia} \mathrm{T}$, et al. Multifunctional inorganic nanoparticles for imaging, targeting, and drug delivery. ACS Nano. 2008; 2: 889-6.

8. Kim S, Lim YT, Soltesz EG, et al. Near-infrared fluorescent type II quantum dots for sentinel lymph node mapping. Nat Biotechnol. 2004; 22: 93-7.

9. Nyk M, Kumar R, Ohulchanskyy TY, et al. High Contrast in vitro and in vivo photoluminescence bioimaging using near infrared to near infrared up-conversion in $\mathrm{Tm} 3+$ and $\mathrm{Yb} 3+$ doped fluoride nanophosphors. Nano Lett. 2008; 8: 3834-8.

10. Deissler V, Ruger R, Frank W, et al. Fluorescent liposomes as contrast agents for in vivo optical imaging of edemas in mice. Small. 2008; 4: 1240-6.

11. Josephson L, Kircher MF, Mahmood U, et al. Near-infrared fluorescent nanoparticles as combined MR/Optical imaging probes. Bioconjugate Chem. 2002; 13: 554-0.

12. He $X$, Nie $H$, Wang $K$, et al. In vivo study of biodistribution and urinary excretion of surface-modified silica nanoparticles. Anal Chem. 2008; 80: 9597-3.

13. Kumar R, Ohulchanskyy TY, Roy I, et al. Near-infrared phosphorescent polymeric nanomicelles: efficient optical probes for tumor imaging and detection. ACS Appl Mater Interfaces. 2009; 1: 1474-1.

14. Torchilin VP. Micellar nanocarriers: pharmaceutical perspectives. Pharm Res. 2007; 24: 1-16.

15. Mohan P, Rapoport N. Doxorubicin as a molecular nanotheranostic agent: effect of doxorubicin encapsulation in micelles or nanoemulsions on the ultrasound-mediated intracellular delivery and nuclear trafficking. Mol Pharm. 2010; 7:1959-73.

16. Krishnadas A, Rubinstein I, Önyüksel H. Sterically stabilized phospholipid mixed micelles: in vitro evaluation as a novel carrier for water-insoluble drugs. Pharm Res. 2003; 20: 297-2.

17. Vakil R, Kwon GS. Effect of cholesterol on the release of amphotericin B from PEG-phospholipid micelles. Mol Pharm. 2008; 5: 98-4.
18. Lukyanov AN, Gao ZG, Mazzola L, et al. Polyethylene glycol-diacyllipid micelles demonstrate increased accumulation in subcutaneous tumors in mice. Pharm Res. 2002; 19: 1424-9.

19. Yong KT, Roy I, Law WC, et al. Synthesis of cRGD-peptide conjugated near-infrared CdTe/ZnSe core-shell quantum dots for in vivo cancer targeting and imaging. Chem Comm. 2010; 46: 7136-8.

20. Cattel L, Ceruti M, Dosio F. From conventional to stealth liposomes: a new frontier in cancer chemotherapy. J Chemother. 2004; 16: 94-7.

21. Frederick CA, Williams LD, Ughetto G, et al. Structural comparison of anticancer drug-DNA complexes: adriamycin and daunomycin. Biochemistry. 1990; 29: 2538-9.

22. Fornari FA, Randolph JK, Yalowich JC, et al. Interference by doxorubicin with DNA unwinding in MCF-7 breast tumor cells. Mol Pharmacol. 1994; 45: 649-6.

23. Cummings J, Smyth JF. DNA topoisomerase I and II as targets for rational design of new anticancer drugs. Ann Oncol. 1993; 4: 533-3.

24. Farokhzad OC, Zhang L, Kantoff P, et al. Quantum dot-aptamer conjugates for synchronous cancer imaging, therapy, and sensing of drug delivery based on bi-flourescence resonance energy transfer. Nano Letters. 2007; 7: 3065-0.

25. Chakravarthy KV, Davidson BA, Helinski JD, et al. Doxorubicin-conjugated quantum dots to target alveolar macrophages and inflammation. Nanomedicine: Nanotechnology, Biology and Medicine. 2011; 7: 88-6.

26. Kim D, Gao ZG, Lee ES, et al. In vivo evaluation of doxorubicin-loaded polymeric micelles targeting folate receptors and early endosomal $\mathrm{pH}$ in drug-resistant ovarian cancer. Mol Pharma. 2009; 6: 1353-2.

27. Patil RR, Guhagarkar SA, Devarajan PV. Engineered nanocarriers of doxorubicin: a current update. Crit Rev Ther Drug Carrier Syst. 2008; 25: $1-61$.

28. Kwon GS. Polymeric micelles for delivery of poorly water-soluble compounds. Crit Rev Ther Drug Carrier Syst 2003; 20: 357-03.

29. Dubertret B, Skourides P, Norris DJ, et al. In vivo imaging of quantum dots encapsulated in phospholipid micelles. Science 2002; 298:1759-2.

30. Carion O, Mahler B, Pons T, et al. Synthesis, encapsulation, purification and coupling of single quantum dots in phospholipid micelles for their use in cellular and in vivo imaging. Nat Protocols 2007; 2: 2383-0.

31. Park JH, Maltzahn G, Ruoslahti E, et al. Micellar Hybrid Nanoparticles for Simultaneous Magneto-Fluorescent Imaging and Drug Delivery. Angew Chem Int Ed. 2008; 47: 7284-8.

32. Ni T, Nagesha DK, Robles J, et al. CdS nanoparticles modified to chalcogen sites: new supramolecular complexes, butterfly bridging, and related optical effects. J Am Chem Soc. 2002; 124: 3980-2.

33. Baker DR, Kamat PV. Tuning the emission of CdSe quantum dots by controlled trap enhancement. Langmuir. 2010; 26: 11272-6.

34. Dabbousi BO, Rodriguez-Viejo J, Mikulec FV, et al. (CdSe)ZnS core-shell quantum dots: synthesis and characterization of a size series of highly luminescent nanocrystallites. J Phys Chem B. 1997; 101: 9463-5.

35. Kumar R, Roy I, Ohulchanskyy TY, et al. Covalently dye-linked, surface-controlled, and bioconjugated organically modified silica nanoparticles as targeted probes for optical imaging. ACS Nano 2008; 2: 449-6. 